\title{
A Color Stability Comparison of Conventional and CAD/CAM Polymethyl Methacrylate Denture Base Materials
}

\section{Usporedba stabilnosti boje konvencionalnih i CAD/CAM polimetilmetakrilata za izradu baze zubne proteze}

\footnotetext{
${ }^{1}$ Program of Dental Technicians, Istanbul Cerrahpasa University, Istanbul, Turkey

Program za dentalne tehničare Sveučilišta Cerrahpasa u Istanbulu, Istanbul, Turska

2 Department of Prosthodontics, Yeni Yüzyıl University Faculty of Dentistry, Istanbul, Turkey

Zavod za stomatološku protetiku Stomatološkog fakulteta Sveučilišta Yeni Yüzyll, Istanbul, Turska

3 Department of Prosthodontics, Istanbul University Faculty of Dentistry, Istanbul, Turkey

Zavod za stomatološku protetiku Stomatološkog fakulteta Sveučilišta u Istanbulu, Istanbul, Turska
}

\section{Abstract}

Objective: The aim of this study was to evaluate the color stability of a heat polymerized resin, an auto-polymerizing resin, a urethane dimethacrylate resin and a CAD/CAM PMMA block resin stored in different storage media. Material and methods: 60 disc-shaped specimens $(15 \times 2 \mathrm{~mm})$ were fabricated for each group. A total of 240 disc-shaped specimens were thermal-cycled for 5,000 cycles. Then specimens were randomized into 4 groups ( $n=15)$ according to storage media: coffee, coke, red wine and distilled water (control group). The color measurement of each sample was performed using a spectrophotometer before and after storage (after 7 and 30 days), and color changes (ĆE) were calculated. Results: All the denture base materials demonstrated dissimilar color changes after stored in the different storage media in both evaluation stages. In all storage media, CAD-CAM denture base resins showed the minimum in color change. In all denture base resins, red wine showed a higher degree of color change than coke or coffee. Conclusion: The color stability of CAD-CAM denture base resins is better than any of the other kind of denture base resins. The color change values of all groups except Eclypse stored in red wine had clinically detectable values.
Received: March 28, 2019

Accepted: June 1, 2019

Address for correspondence

Cagatay Dayan

Istanbul Cerrahpasa University Program of Dental Technicians Istanbul, Turkey

suleyman.dayan@istanbul.edu.tr

Key words

PMMA, CAD-CAM Acrylic Resin, Discoloration, Denture Base Resin

\section{Introduction}

Polymethyl methacrylate (PMMA) resin has been successfully used for denture base materials for years $(1,2)$. Several different kinds of denture base acrylic resins are used and named according to their production mode, such as auto-polymerizing acrylic resin, heat-polymerized acrylic resin, light cure resin, special form resins polymerized in microwave processing and computer-aided design/computer aided manufacturing (CAD/CAM) PMMA block resin $(3,4)$. These materials have many advantages, including their low cost, ease of manipulation, adequate mechanical and physical properties and satisfactory appearance. Despite these advantages, the following disadvantages exist for PMMA: hypersensitivity, color change, abrasion, and porosity $(5,7)$.

Light-activated urethane dimethacrylate resins were developed to replace PMMA for eliminate long flasking procedure and contact allergies. Recently, a new light-activated denture base material named Eclypse (Eclipse, Dentsply, York, PA, USA) has been introduced. The denture base record is produced from the baseplate resin, and after light polymerization it becomes the permanent denture base of the
Uvod

Polimetilmetakrilatna (PMMA) smola već se godinama uspješno primjenjuje kao materijal za izradu baze proteza $(1,2)$. Upotrebljava se nekoliko različitih vrsta akrilatnih smola koje se dijele prema načinu polimerizacije, na primjer, autopolimerizirajuća akrilatna smola, akrilatna smola polimerizirana toplinom, akrilatna smola polimerizirana svjetlošću, posebna smola polimerizirana u mikrovalnoj obradi i PMMA smola za računalno potpomognutu proizvodnju (CAD/CAM) koja dolazi u blokovima $(3,4)$. Uključujući male troškove, jednostavnost rukovanja, odgovarajuća mehanička i fizička svojstva i zadovoljavajući izgled, ti materijali imaju mnoge prednosti. Unatoč tomu, PMMA ima i nedostatke - preosjetljivost, promjenu boje, abraziju i poroznost $(5,7)$.

Proizvedene su svjetlosno polimerizirajuće uretanske dimetakrilatne smole koje zamjenjuju PMMA-u kako bi se izbjegao postupak dugotrajnog ulaganja i kontaktne alergije. Posljednjih godina pojavio se novi materijal za izradu baze proteze koji se aktivira svjetlošću i zove se Eclypse (Eclipse, Dentsply, York, PA, SAD). Registrat za bazu proteze dobiva se iz ploče od smole i nakon svjetlosne polimerizacije ona po- 
final denture. Eclipse denture base has showed significantly higher impact and flexural strength when compared to PMMA denture bases (8).

In recent years, with advancements in CAD/CAM technology, manufacturers have produced CAD/CAM PMMA based polymer blocks as an alternative for denture base resins $(4,9)$. CAD/CAM PMMA block manufacturers claim that these materials will have better mechanical properties than conventional denture base resins (10). CAD/CAM PMMA based polymer blocks, that are polymerized under high temperature and high pressure conditions, reduce residual monomer release, improve optical properties, improve stability of color and facilitate the production of denture bases by easy milling $(11,12)$.

The appearance and color of denture base is an important property of the denture. In addition, denture base material should match the color and appearance of the underlying tissues (1). One of the most important clinical features of all dental materials is color stability and any color changes are indicators of aging or damaged materials (13-15). Additionally, the esthetic appearance of the prosthesis is one of the important factors in meeting the expectations of patients (16-18).

Various factors may affect the color change of denture base materials after prolonged use. These factors are: water absorption, stain accumulation, degradation of intrinsic pigments, dissolution of ingredients, foods, beverages and roughness of surface $(1,19,20)$.

When assessing color alterations, visual examination is a subjective physiological and psychological procedure. On the contrary, when the spectrophotometer is used for a determination of color alteration, not only does it eliminate subjective interpretations but also allows identification of minor color alterations (21). A color system the name of which is The Commission Internationale de l'Eclairage (CIE) $\mathrm{L}^{*} \mathrm{a}^{*} \mathrm{~b}$ is a constant color scale that includes all the colors visible to the human eye. Hence, it is appropriate for perceptual studies of color changes in dental materials (22).

Even though the current scientific data promote $\mathrm{CAD} /$ CAM- fabricated complete dentures clinical superiority, the data about their material properties are still limited (23). Therefore, the aim of the present study was to compare the influence of various storage media on the color changes of an autopolymerizing resin, heat polymerized resin, urethane dimethacrylate resin and a CAD/CAM PMMA block resin. The null hypothesis was that that different storage media does not affect the color changes in various denture base resins.

\section{Material and Methods}

Four kinds of denture base resins were used in this study; an auto-polymerizing resin (A), a heat polymerized resin $(\mathrm{H})$, a light-activated resin $(\mathrm{L})$ and a CAD/CAM block resin $(\mathrm{C})$. All of the materials were used according to the manufacturer's recommended procedures. The denture base resins used in this study and their manufacturers are summarized in Table 1 .

Sixty disc-shaped specimens were prepared for each group. The CAD/CAM PMMA denture base materials were staje baza konačne proteze. Eclypse ima znatno veću udarnu i savojnu čvrstoću u usporedbi s bazama od PMMA-e (8).

Posljednjih godina, s napretkom u CAD/CAM tehnologije, proizvođači izrađuju polimerne blokove na bazi PMMAe kao alternativu smolama za izradu baze proteze $(4,9)$. Proizvođači CAD/CAM PMMA blokova tvrde da ti materijali imaju bolja mehanička svojstva od konvencionalnih smola (10). Blokovi PMMA-e za CAD/CAM obradu koji se polimeriziraju u uvjetima visoke temperature i tlaka, manje otpuštaju zaostatni monomer, imaju bolja optička svojstva, bolju stabilnost boje i omogućuju izradu baze proteze jednostavnim glodanjem $(11,12)$.

Izgled i boja baze važna su svojstva proteze. $\mathrm{Uz}$ to, baza proteze bojom i izgledom treba oponašati okolna tkiva (1). Jedna od najvažnijih kliničkih značajki svih stomatoloških materijala jest postojanost boje, pa su bilo kakve promjene toga svojstva pokazatelji starenja ili oštećenja materijala (13 - 15). Osim toga, estetski izgled proteze jedan je od važnih čimbenika u ispunjavanju očekivanja pacijenata (16 - 18).

Različiti čimbenici mogu utjecati na promjenu boje materijala za bazu proteze nakon duljeg korištenja. Među njima su upijanje vode, nakupljanje mrlja, degradacija intrinzičnih pigmenata, otapanje sastojaka, hrana, piće i hrapavost površine $(1,19,20)$.

Pri procjeni promjene boje, vizualni pregled je subjektivni fiziološki i psihološki postupak. Ali kada se spektrofotometar rabi za određivanje promjene boje, taj uređaj ne samo da eliminira subjektivno tumačenje, nego omogućuje i prepoznavanje manjih promjena boje (21). Sustav boja koji je definirao Commission Internationale de l'Eclairage kao (CIE) $\mathrm{L}^{*} \mathrm{a}^{*} \mathrm{~b}$, konstantna je ljestvica boja koja uključuje sve nijanse vidljive ljudskom oku. Zato je prikladan za perceptivna istraživanja promjene boje dentalnih materijala (22).

Iako aktualni znanstveni podatci govore u prilog kliničkoj nadmoći potpunih proteza izrađenih CAD/CAM tehnologijom, oni o svojstvima materijala još su ograničeni (23). Zato je svrha ovog istraživanja bila usporediti utjecaj različitih medija na promjene boje autopolimerizirajuće smole, toplinski polimerizirajuće smole, uretan-dimetakrilatne smole i CAD/ CAM PMMA bloka. Nulta hipoteza glasila je da različiti mediji za pohranu ne utječu na promjenu boje različitih smola za izradu baze proteze.

\section{Materijali i metode}

U ovom istraživanju upotrijebljene su četiri vrste smola za izradu baze proteze - autopolimerizirajuća smola (A), toplinski polimerizirajuća smola $(\mathrm{H})$, svjetlosno polimerizirajuća smola (L) i CAD/CAM blok od smole (C). Svi materijali upotrebljeni su prema preporukama proizvođača. Smole za izradu bazu proteze korištene u ovom istraživanju i njihovi proizvođači sažeti su u tablici 1 .

Za svaku skupinu pripremljeno je 60 uzoraka u obliku pločica. CAD/CAM PMMA materijali dizajnirani su u sof- 
Table 1 Materials and manufacturer information

Tablica 1. Materijali i podatci o proizvođaču

\begin{tabular}{|c|c|c|c|}
\hline $\begin{array}{l}\text { Acrylic resin } \\
\text { Akrilatna smola }\end{array}$ & $\begin{array}{l}\text { Symbol } \\
\text { Simbol }\end{array}$ & $\begin{array}{l}\text { Product name } \\
\text { Naziv proizvoda }\end{array}$ & Manufacturer $\bullet$ Proizvođač \\
\hline $\begin{array}{l}\text { Autopolymerizing resin } \bullet \\
\text { Autopolimerizirajuća smola }\end{array}$ & A & Weropress 1/218-05, Monomer 32215 & $\begin{array}{l}\text { Merz Dental GmbH, Lütjenburg, } \\
\text { Gemany } \bullet \text { Njemačka }\end{array}$ \\
\hline $\begin{array}{l}\text { Heat polymerized resin } \bullet \\
\text { Toplinski polimerizirajuća smola }\end{array}$ & $\mathrm{H}$ & Paladent 20 K0101103 & $\begin{array}{l}\text { Kulzer Mitsui Chemicals Group, Hanau, } \\
\text { Gemany • Njemačka }\end{array}$ \\
\hline $\begin{array}{l}\text { Light-activated resin } \bullet \\
\text { Svjetlosno polimerizirajuća smola }\end{array}$ & $\mathrm{L}$ & Eclipse 110929 & $\begin{array}{l}\text { Dentsply, Surrey, } \\
\text { United Kingdom • Ujedinjeno Kraljevstvo }\end{array}$ \\
\hline $\begin{array}{l}\text { CAD/CAM block resin } \bullet \\
\text { CAD/CAM blok smole }\end{array}$ & $\mathrm{C}$ & M-PM Disc 44317 & $\begin{array}{l}\text { Merz Dental GmbH, Lütjenburg, } \\
\text { Gemany・Njemačka }\end{array}$ \\
\hline
\end{tabular}

designed as STL files and milled by CAD-CAM milling system (Ceramill Motion 2; Amann Girrbach). Then specimens were sliced with a cutting machine (IsoMet 1000; Buehler) and diamond-wafering blade (IsoMet Blade 15 LC; Buehler) to obtain disc-shaped specimens $2 \mathrm{~mm}$ in thickness. Previously prepared CAD/CAM specimens were coated with high viscosity polyvinylsiloxane (Silagum Putty, DMG, Hamburg, Germany). They were first invested in conventional flasks by Type 3 dental stone (Moldano; Heraeus Kulzer, Hanau, Germany). Acrylic resins were mixed and applied in according to the manufacturers' instructions. Heat polymerized acrylic specimens (Paladent 20) were polymerized at $74^{\circ} \mathrm{C}$ for 9 hours in the automatic polymerization unit (Kavo EWL 5501, Kavo Electrotechnisches Werk GmbH, Germany). Polymerizations of Weropress specimens were performed in a pressure pot heat cure unit (Ivomat IP3, Ivoclar Vivadent AG, Schaan, Lichtenstein) at $45^{\circ} \mathrm{C}$ for 12 minutes under pressure of 2 bars.

A Teflon mold was designed with a transparent Plexiglas lid to produce the Eclipse specimens. The Eclipse specimens were cured in specific unit (Enterra VLC Curing Unit; DeguDent $\mathrm{GmbH}$, Hanau, Germany) using 15-minute polymerization cycle. The polymerization residue materials were then removed with tungsten carbide burs using a handpiece at low speed. Smoothing process was used with a 400 -grit silicon carbide abrasive paper (English Abrasives) on a machine (Phoenix Beta; Buehler). The specimens were polished by a conventional pre-polishing technique using slurry of coarse pumice (IMIPOMZA; Imıcryl), water and a bristle brush on a polishing lathe (P1000; Zubler) at a rate of $1500 \mathrm{rpm}$ for 90 seconds. Polishing process was conducted with a conventional pre-polishing technique using a bristle brush on the rough pumice water slurry and a polishing lathe at a speed of $1500 \mathrm{rpm}$ at $90 \mathrm{rpm}$ and then fine polishing were done with using a polishing paste (chalk plus alcohol) and lathe flannel wheel for 90 seconds. All specimens were ultrasonically cleaned (Araysonic; Array) in distilled water for 10 minutes and dried with a paper towel.

A total of 240 disc-shaped specimens were thermal cycled for 5,000 cycles between $5^{\circ} \mathrm{C}$ and $55^{\circ} \mathrm{C}$ with a 30 -second dwell time and a 20-second transfer time from one bath to the other. The specimens were divided into four main groups $(\mathrm{n}=15)$, and each group was divided into 4 subgroups $(\mathrm{n}=10)$ according to storage media: coffee, coke, red wine and distilled water. The distilled water group was used as a control. tveru i kao STL datoteke obrađeni u glodalici (Ceramill Motion 2; Amann Girrbach, SAD). Zatim su rezani strojno (IsoMet 1000; Buehler) dijamantnom oštricom (IsoMet Blade 15 LC; Buehler, SAD) kako bi se dobili uzorci u obliku pločica debljine $2 \mathrm{~mm}$. Pripremljeni CAD/CAM uzorci obloženi su polivinil-siloksanom visoke viskoznosti (Silagum Putty, DMG, Hamburg, Njemačka), nakon ulaganja u konvencionalne kivete koristeći se sadrom tipa 3 (Moldano; Heraeus Kulzer, Hanau, Njemačka). Akrilatne smole zamiješane su pripremljene prema uputama proizvođača. Toplinski polimerizirajući akrilatni uzorci (Paladent 20) polimerizirani su 9 sati na $74^{\circ} \mathrm{C}$ u automatskoj polimerizacijskoj jedinici (Kavo EWL 5501, Kavo Electrotechnisches Werk GmbH, Njemačka). Polimerizacija uzoraka Weropress obavljena je u Ivomatu (Ivomat IP3, Ivoclar Vivadent AG, Schaan, Lihtenštajn) na temperaturi od $45^{\circ} \mathrm{C}$ tijekom 12 minuta i pod tlakom od 2 bara.

Za izradu uzoraka Eclypse izrađen je teflonski kalup s prozirnim poklopcem od pleksiglasa. Uzorci su polimerizirani u posebnoj jedinici (Enterra VLC Curing Unit; DeguDent $\mathrm{GmbH}$, Hanau, Njemackka) primjenom 15-minutnog ciklusa polimerizacije. Površina je obrađena karbidnim brusnim sredstvom pri maloj brzini. Zatim je strojno očišćena abrazivnim papirom od silicijeva karbida gruboće 400 (Phoenix Beta; Buehler). Uzorci su polirani konvencionalnom tehnikom pretpoliranja 90 sekunda koristeći se kašom od krupnog plovučca (IMIPOMZA; Imıcryl), vodom i četkom na stroju za poliranje (P1000; Zubler), a brzina je bila 1500 okretaja u minuti. Proces poliranja obavljen je konvencionalnom tehnikom pretpoliranja s pomoću četkice i grube vodene suspenzije plovučća pri brzini od 1500 okretaja u minuti tijekom 90 sekunda, a zatim finim poliranjem pastom za poliranje (kreda plus alkohol) i kožnim polirerom također 90 sekunda. Svi uzorci čišćeni su ultrazvučno (Araysonic; Aray) u destiliranoj vodi 10 minuta i osušeni papirnatim ručnikom.

Ukupno 240 uzoraka u obliku pločica izloženo je termocikliranju od 5000 ciklusa između $5{ }^{\circ} \mathrm{C}$ i $55^{\circ} \mathrm{C}$ s vremenom zadržavanja od 30 sekunda i 20 -sekundnim prijenosom iz jedne kupke u drugu. Uzorci su podijeljeni u četiri glavne skupine $(\mathrm{n}=15)$, a svaka je razvrstana $\mathrm{u}$ isto toliko podskupina $(\mathrm{n}=10)$ prema mediju za pohranu: kava, kola, crno vino i destilirana voda. Skupina s destiliranom vodom korištena je kao kontrola.

Svi postupci provedeni su na $23^{\circ} \mathrm{C}$ i to je radio isti istraživač kako bi se osigurala standardizacija i izbjegle varijacije 
All process steps were accomplished by the same operator at $23^{\circ} \mathrm{C}$ room temperature to ensure standardization and avoid change due to temperature.

All operations were carried out at $23^{\circ} \mathrm{C}$ to ensure standardization by the same operator and to avoid changes due to temperature. In accordance with the recommendations of the coffee manufacturer, a coffee solution was prepared by mixing $15 \mathrm{~g}$ of instant coffee powder (Nescafé Classic; Nestlé, Vevey, Switzerland; pH 5.56) with $200 \mathrm{~mL}$ of hot water and sugar free. After the preparation, the coffee solution was allowed to cool down to room temperature. There was no special preparation for the coke (Coca Cola Co, Atlanta, GA; $\mathrm{pH}$ 2.37) or red wine (Vinkara Winehouse, Ankara, Turkey; $\mathrm{pH}$ 3.6) groups. The specimens were kept in storage media for 15 minutes twice per day, the solution media were refreshed on a daily basis for up to a 30 days. The $\mathrm{pH}$ values of the storage media were verified by a $\mathrm{pH}$ meter (HI 221; Hanna Instruments Inc., Woonsocket, RI) before each storage. After the storage periods had been completed, the specimens were washed with and then stored in distilled water. This procedure was followed for 30 days. The specimens were kept in distilled water at $37^{\circ} \mathrm{C}$ between storage periods.

The color data was recorded before and after storage $(7$ and 30 days) in different media according to the CIE L*a*b* color scale using a spectrophotometer (Data color CHECK 3 , USA). The color difference $(\Delta \mathrm{E})$ between the color coordinates was calculated by applying the formula $\Delta \mathrm{E}^{*}=\left[\left(\Delta \mathrm{L}^{*}\right) 2+\right.$ $\left.\left(\Delta \mathrm{a}^{*}\right) 2+\left(\Delta \mathrm{b}^{*}\right) 2\right] 1 / 2$ in order to compare values before and after the storage treatment. Each sample was subjected to color measurement four times and the average value was recorded.

\section{Statistical Analyses}

Statistical analysis was performed using Statistical Package for Social Sciences (SPSS) for Windows software (IBM Corp. Released 2013.The IBM SPSS Statistics for Windows, Version 22.0. Armonk, NY, USA). The Shapiro-Wilk test was used to identify if the measured parameters met the assumptions of normal distribution. The results showed that the data were not normally distributed. Between the groups, the color differences $(\Delta \mathrm{E})$ were analyzed by the Kruskal- Wallis test at 7-day and 30-day periods, while median and range values were used in the descriptive statistical analysis. Between the groups, multiple comparisons were performed using the Mann-Whitney U test.

\section{Results}

Table 2 shows the color change values after storage in the four different storage media for four denture base materials after the period of 7 and 30 days. The Weropress denture base resins demonstrated the highest color change in red wine, which represents a significant difference when compared to the other storage media both at 7 and 30 days $(\mathrm{p}<0.001)$. The Paladent denture base resins demonstrated the highest color change in distilled water, which represents a significant difference when compared to the other storage media after 7 days, after 30 days it demonstrated the highest color change in red wine, which represents a significant difference when compared to the other storage media $(\mathrm{p}<0.001)$. The Eclypse den- zbog promjene temperature. U skladu s uputama proizvodača, otopina kave pripremljena je miješanjem $15 \mathrm{~g}$ praha instantne kave (Nescafé Classic; Nestlé, Vevey, Švicarska; pH $5,56)$ s $200 \mathrm{ml}$ vruće vode i bez šećera. Nakon što je pripremljena, otopina kave ostavljena je da se ohladi na sobnu temperaturu. Nije bilo posebne pripreme za kolu (Coca Cola Co, Atlanta, GA, SAD; pH 2,37) ili za crno vino (Vinkara Winehouse, Ankara, Turska; pH 3,6). Uzorci su čuvani u mediju za pohranjivanje po 15 minuta dva puta na dan, a otopina je osvježavana svaki dan tijekom 30 dana. Vrijednosti $\mathrm{pH}$ medija za pohranjivanje potvrđene su $\mathrm{pH}$-metrom (HI 221; Hanna Instruments Inc, Woonsocket, RI, SAD) prije svakog uranjanja. Nakon završstka pohranjivanja uzorci su isprani i odloženi u destiliranu vodu. Taj je postupak ponavljan $30 \mathrm{da}$ na, a uzorci su, između pohranjivanja u obojenim otopinama, držani u destiliranoj vodi na temperaturi od $37^{\circ} \mathrm{C}$.

Podatci o boji zabilježeni su prije skladištenja i nakon toga postupka (7 i 30 dana) u različitim medijima prema CIE L*a*b* ljestvici s pomoću spektrofotometra (Data color CHECK 3, SAD). Razlika u boji $(\triangle \mathrm{E})$ između koordinata boja izračunata je primjenom jednadžbe $\Delta \mathrm{E}^{*}=\left[\left(\Delta \mathrm{L}^{*}\right) 2\right.$ $\left.+\left(\Delta \mathrm{a}^{*}\right) 2+\left(\Delta \mathrm{b}^{*}\right) 2\right] 1 / 2$, kako bi se usporedile vrijednosti prije pohranjivanja u tekućinama i poslije toga postupka. Svaki uzorak podvrgnut je mjerenju boje četiri puta i zabilježena je prosječna vrijednost.

\section{Statistička analiza}

Statistička analiza obavljena je Windowsovim statističkim paketom za društvene znanosti (SPSS) (IBM Corp. 2013. IBM SPSS Statistics for Windows, verzija 22.0. Armonk, NY, SAD). Shapiro-Wilkov test primijenjen je za utvrđivanje jesu li izmjereni parametri zadovoljili pretpostavke normalne distribucije. Rezultati su pokazali da podatci nisu bili normalno distribuirani. Razlike između skupina $(\Delta \mathrm{E})$ analizirane su Kruskal-Wallisovim testom nakon 7 i 30 dana, a vrijednosti medijana i rankovi korištene su u deskriptivnoj statističkoj analizi. Između skupina provedene su višestruke parne usporedbe uporabom Mann-Whitneyeva U-testa s razinom značajnosti od 0,05 .

\section{Rezultati}

Tablica 2. pokazuje da su se vrijednosti boje svih četiriju materijala za bazu proteze mijenjale nakon pohranjivanja u četirima različitim medijima nakon 7 i 30 dana. Smola za bazu proteze Weropress pokazala je najveću promjenu boje u crnom vinu, što je značajna razliku u usporedbi s drugim medijima za pohranu nakon 7 i 30 dana $(\mathrm{p}<0,001)$. Smola za bazu proteze Paladent imala je najveću promjenu boje u destiliranoj vodi, što je značajna razliku u usporedbi s drugim medijima za pohranu nakon 7 dana. Nakon 30 dana pokazala je najveću promjenu boje u crnom vinu i to je značajna razliku u usporedbi s ostalim medijima za pohranu $(\mathrm{p}<0,001)$. Eclypse smola za bazu proteze pokazala je najveću promje- 
Table 2 Different denture base resins after immersion in different solutions at 1 week and 1 month.

Tablica 2. Smole za izradu baze proteze nakon uranjanja u različite otopine nakon jednog tjedna i jednog mjeseca

\begin{tabular}{|c|c|c|c|c|c|c|c|}
\hline \multirow{2}{*}{ Material • Materijal } & \multirow[b]{2}{*}{ Solutions • Otopina } & \multicolumn{3}{|c|}{1 Week $\bullet 1$ tjedan } & \multicolumn{3}{|c|}{1 Month $\bullet 1$ mjesec } \\
\hline & & $\begin{array}{l}\text { Mean } \\
\text { Rank • } \\
\text { Srednji } \\
\text { rankovi }\end{array}$ & $\begin{array}{l}\text { Median } \\
\text { (Range) • } \\
\text { Medijan }\end{array}$ & $\begin{array}{l}\text { Mann- } \\
\text { Whitney } \\
\text { U test• } \\
\text { Mann- } \\
\text { Whitneyev } \\
\text { U-test }\end{array}$ & $\begin{array}{l}\text { Mean } \\
\text { Rank } \\
\text { Srednji } \\
\text { rankovi }\end{array}$ & $\begin{array}{l}\text { Median } \\
\text { (Range) • } \\
\text { Medijan }\end{array}$ & $\begin{array}{l}\text { Mann- } \\
\text { Whitney } \\
\text { U test• } \\
\text { Mann- } \\
\text { Whitneyev } \\
\text { U-test }\end{array}$ \\
\hline \multirow{4}{*}{ Weropress } & a - Coffee $\bullet$ Kava & 50.07 & $0.97(0.22)$ & b.c $>$ a & 57.36 & $1.99(0.16)^{a}$ & $c>a \cdot b$ \\
\hline & b - Coke $\bullet$ Kola & 66.07 & $0.99(0.05)$ & & 58.64 & $2.00(0.14)^{\mathrm{a}}$ & \\
\hline & $\mathrm{c}$ - Red Wine $\bullet$ Crno vino & 79.18 & $1.00(0.08)$ & & 95.50 & $2.45(0.59)^{\mathrm{b}}$ & \\
\hline & $\begin{array}{l}\mathrm{d} \text { - Distilled Water } \\
\text { Destilirana voda }\end{array}$ & 30.68 & $0.94(0.74)$ & $a>d$ & 14.50 & $0.90(0.82)^{\mathrm{c}}$ & $a \cdot b>d$ \\
\hline $\begin{array}{l}\text { Kruskal-Wallis test. p value } \bullet \\
\text { Kruskal-Wallisov test. } \text { - } \text {-vrijednost }\end{array}$ & & & 0.001 & & & 0.001 & \\
\hline \multirow{4}{*}{ Paladent } & a - Coffee $\cdot$ Kava & 50.00 & $1.02(0.06)$ & $a . b>c$ & 55.39 & $1.92(0.19)$ & $a \cdot b<c$ \\
\hline & b - Coke $\bullet$ Kola & 53.04 & $1.02(0.07)$ & & 61.71 & $1.92(0.44)$ & \\
\hline & c- Red Wine • Crno vino & 34.00 & $1.00(0.08)$ & & 94.39 & $2.17(0.66)$ & \\
\hline & $\begin{array}{l}\mathrm{d} \text { - Distilled Water } \\
\text { Destilirana voda }\end{array}$ & 88.96 & $1.06(0.24)$ & $d>a . b$ & 14.50 & $1.04(0.14)$ & a.b>d \\
\hline $\begin{array}{l}\text { Kruskal-Wallis test. p value } \bullet \\
\text { Kruskal-Wallisov test. p-vrijednost }\end{array}$ & & & 0.001 & & & 0.001 & \\
\hline \multirow{4}{*}{ Eclipse } & a - Coffee $\bullet$ Kava & 90.36 & $1.08(0.57)$ & $a>c$ & 69.54 & $3.02(1.08)$ & $a>b$ \\
\hline & b - Coke $\bullet$ Kola & 28.82 & $0.94(0.15)$ & & 43.46 & $2.90(0.96)$ & $b>d$ \\
\hline & $\mathrm{c}$ - Red Wine $\bullet$ Crno vino & 73.86 & $1.04(0.14)$ & $c>b . d$ & 98.50 & $3.59(1.32)$ & $\mathrm{c}>\mathrm{a}$ \\
\hline & $\begin{array}{l}\mathrm{d} \text { - Distilled Water } \\
\text { Destilirana voda }\end{array}$ & 32.96 & $0.96(0.15)$ & & 14.50 & $1.18(0.96)$ & \\
\hline $\begin{array}{l}\text { Kruskal-Wallis test. p value } \bullet \\
\text { Kruskal-Wallisov test. p-vrijednost }\end{array}$ & & & 0.001 & & & 0.001 & \\
\hline \multirow{4}{*}{ Cad-Cam Acrylic } & a - Coffee $\bullet$ Kava & 71.14 & $0.76(0.16$ & $a>b$ & 68.61 & $1.08(0.27)$ & $a>b$ \\
\hline & b - Coke $\bullet$ Kola & 40.93 & $0.53(0.13)$ & $b>d$ & 44.39 & $0.99(0.29)$ & $b>d$ \\
\hline & $\mathrm{c}$ - Red Wine • Crno vino & 97.86 & $0.90(0.50)$ & $\mathrm{c}>\mathrm{a}$ & 98.50 & $2.04(1.03)$ & $\mathrm{c}>\mathrm{a}$ \\
\hline & $\begin{array}{l}\mathrm{d} \text { - Distilled Water } \\
\text { Destilirana voda }\end{array}$ & 16.07 & $0.44(0.19)$ & & 14.50 & $0.43(0.64)$ & \\
\hline $\begin{array}{l}\text { Kruskal-Wallis test. p value } \bullet \\
\text { Kruskal-Wallisov test. p-vrijednost }\end{array}$ & & & 0.001 & & & 0.001 & \\
\hline
\end{tabular}

ture base resins demonstrated the highest color change in coffee, which represents a significant difference when compared to the other storage media after 7 days, after 30 days it showed the highest color change in red wine, which represents a significant difference when compared to the other storage media $(\mathrm{p}<0.001)$. The CAD-CAM acrylic denture base resins demonstrated the highest color change in red wine, which represents a significant difference when compared to the other storage media at both 7 and 30 days $(\mathrm{p}<0.001)$.

Table 3 shows the discoloration effects of storage media on the denture base resins after 7 and 30 days. The coffee solution, 7 and 30 days, affected the Eclypse denture base the most $(\mathrm{p}<0.001)$. The coke solution affected the Paladent denture base the most after 7 days, at the end of 30 days the Eclypse denture base was the most affected $(p<0.001)$. The red wine solution affected the Eclypse denture base the most both at 7 and 30 days $(p<0.001)$. The distilled water affected the Paladent denture base the most 7 days, at the end of 30 days the Eclypse denture base was the most effected $(\mathrm{p}<0.001)$. nu boje u kavi, što je značajna razlika u usporedbi s drugim medijima za pohranu nakon 7 dana. Nakon 30 dana pokazala je najveću promjenu boje u crnom vinu, što je značajna razlika u usporedbi s drugim medijima za pohranu ( $\mathrm{p}<$ 0,001). CAD/CAM materijali za bazu proteze imali su najveću promjenu boje u crnom vinu, što je značajna razlika u usporedbi $s$ drugim medijima za pohranu nakon 7 i 30 dana $(\mathrm{p}<0,001)$.

U tablici 3. nalaze su učinci diskoloracije medija za pohranu na smole za izradu baze proteze nakon 7 i 30 dana. Otopina kave nakon 7 i 30 dana najviše je utjecala na materijal za bazu proteze Eclypse ( $\mathrm{p}<0,001)$. Otopina kole najviše je utjecala na bazu proteze Paladent nakon 7 dana, a nakon 30 dana najveća promjena zabilježena je na materijalu Eclypse $(\mathrm{p}<0,001)$. Otopina crvenoga vina najviše je utjecala na bazu proteze Eclypse i nakon $7 \mathrm{i}$ nakon 30 dana $(\mathrm{p}<0,001)$. Destilirana voda najsnažnije je djelovala na materijal Paladent nakon 7 dana, a nakon 30 dana najveća promjena zabilježena je na Eclypseu $(\mathrm{p}<0,001)$. 
Table 3 Different solutions on denture base materials at 1 week and 1 month.

Tablica 3. Različite otopine na materijalima za bazu proteze nakon jednog tjedna i jednog mjeseca

\begin{tabular}{|c|c|c|c|c|c|c|c|}
\hline \multirow[b]{2}{*}{ Solutions $\bullet$ Otopina } & \multirow[b]{2}{*}{ Material $\bullet$ Materijal } & \multicolumn{3}{|c|}{1 Week $\bullet 1$ tjedan } & \multicolumn{3}{|c|}{1 Month $\bullet 1$ mjesec } \\
\hline & & $\begin{array}{l}\text { Mean } \\
\text { Rank• } \\
\text { Srednji } \\
\text { rankovi }\end{array}$ & $\begin{array}{l}\text { Median } \\
\text { (Range) } \\
\text { Medijan }\end{array}$ & $\begin{array}{c}\text { Mann- } \\
\text { Whitney } \\
\text { U test } \\
\text { Mann- } \\
\text { Whitneyev } \\
\text { U-test }\end{array}$ & $\begin{array}{l}\text { Mean } \\
\text { Rank• } \\
\text { Srednji } \\
\text { rankovi }\end{array}$ & $\begin{array}{l}\text { Median } \\
\text { (Range) • } \\
\text { Medijan }\end{array}$ & $\begin{array}{c}\text { Mann- } \\
\text { Whitney } \\
\text { U test } \\
\text { Mann- } \\
\text { Whitneyev } \\
\text { U-test }\end{array}$ \\
\hline \multirow{4}{*}{ Coffee $\bullet$ Kava } & a - Weropress & 47.00 & $0.97(0.22)$ & $a>d$ & 68.75 & $1.99(0.16)$ & $a>d$ \\
\hline & b - Eclypse & 93.32 & $1.08(0.57)$ & $\mathrm{b}>\mathrm{c}$ & 9.25 & $3.02(1.08)$ & $\mathrm{b}>\mathrm{c}$ \\
\hline & c-Paladent & 71.18 & $1.02(0.06)$ & $\mathrm{c}>\mathrm{a}$ & 44.50 & $1.92(0.19)$ & $\mathrm{c}<\mathrm{a}$ \\
\hline & d - Cad-Cam Acrylic & 14.50 & $0.76(0.16)$ & $\mathrm{d}<\mathrm{c}$ & 14.50 & $1.08(0.27)$ & $\mathrm{d}<\mathrm{c}$ \\
\hline $\begin{array}{l}\text { Kruskal-Wallis test. p value } \\
\text { Kruskal-Wallisov test. p-vrijednost }\end{array}$ & & & 0.001 & & & 0.001 & \\
\hline \multirow{3}{*}{ Coke $\bullet$ Kola } & a - Weropress & 65.18 & $0.99(0.05)$ & $a>b$ & 66.64 & $2.00(0.14)$ & $\mathrm{a}<\mathrm{b}$ \\
\hline & c-Paladent & 97.36 & $1.02(0.07)$ & $\mathrm{c}>\mathrm{a}$ & 46.61 & $1.92(0.44)$ & $\mathrm{c}<\mathrm{a}$ \\
\hline & d - Cad-Cam Acrylic & 14.50 & $0.53(0.13)$ & & 14.50 & $0.99(0.29)$ & $\mathrm{d}<\mathrm{c}$ \\
\hline $\begin{array}{l}\text { Kruskal-Wallis test. p value } \\
\text { Kruskal-Wallisov test. p-vrijednost }\end{array}$ & & & 0.001 & & & 0.001 & \\
\hline \multirow{4}{*}{ Red Wine $\bullet$ Crno vino } & a - Weropress & 56.64 & $1.00(0.08)$ & a.c $>d$ & 57.25 & $2.45(0.59)$ & $\mathrm{a}<\mathrm{b}$ \\
\hline & b - Eclypse & 85.36 & $1.04(0.14)$ & $\mathrm{b}>\mathrm{a} . \mathrm{c}$ & 98.50 & $3.59(1.32)$ & \\
\hline & c- Paladent & 65.71 & $1.00(0.08)$ & & 38.46 & $2.17(0.66)$ & c. $\mathrm{d}<\mathrm{a}$ \\
\hline & d - Cad-Cam Acrylic & 18.29 & $0.90(0.50)$ & & 31.79 & $2.04(1.03)$ & \\
\hline $\begin{array}{l}\text { Kruskal-Wallis test. p value } \bullet \\
\text { Kruskal-Wallisov test. p-vrijednost }\end{array}$ & & & 0.001 & & & 0.001 & \\
\hline \multirow{3}{*}{ Distilled Water • Destilirana voda } & a - Weropress & 54.00 & $0.94(0.74)$ & $\mathrm{a} \cdot \mathrm{b}<\mathrm{c}$ & 44.36 & $0.90(0.82) \mathrm{A}$ & $\mathrm{a}<\mathrm{c}$ \\
\hline & b - Eclypse & 60.18 & $0.96(0.15)$ & & 93.00 & $1.18(0.96) \mathrm{B}$ & $\mathrm{b}>\mathrm{c}$ \\
\hline & c- Paladent & 97.32 & $1.06(0.24)$ & & 73.68 & $1.04(0.14) \mathrm{C}$ & \\
\hline $\begin{array}{l}\text { Kruskal-Wallis test. p value } \bullet \\
\text { Kruskal-Wallisov test. p-vrijednost }\end{array}$ & & & 0.001 & & & 0.001 & \\
\hline
\end{tabular}

\section{Discussion}

In the current study, the previously introduced $\mathrm{CAD} /$ CAM PMMA block resins were compared with an autopolymerizing resin, a conventional heat polymerized resin, a urethane dimethacrylate resin, as storage media using red wine, coke, and coffee because basically that these beverages are frequently consumed by people. The denture base materials which were tested showed significantly different discoloration after storage in the different media at both evaluation stages. Hence, the null hypothesis of the study was rejected.

A color change that is more than detectable $(\Delta \mathrm{E}>1.0)$ is considered acceptable up to a $\Delta \mathrm{E}$ value of 3,3 in dentistry; above this value it is considered unacceptable $(24,25)$. Only the Eclypse denture base groups, stored in red wine, showed that the discoloration was clinically unacceptable after the 30 days $(\triangle \mathrm{E} 3,59)$.

The color change was determined for all the acrylic denture base resins and they all increased over time. There are intrinsic and extrinsic factors that can cause discoloration of denture base materials $(15,26)$. These factors include: physical chemical change, stain accumulation, the residual monomer used, water absorption and, degradation of intrinsic pigments, dissolution of the ingredients and the surface roughness. It is well known that beverages such as coffee,

\section{Rasprava}

U ovom istraživanju CAD/CAM PMMA blokovi od smole uspoređeni su s autopolimerizirajućom smolom, konvencionalnom toplinski polimerizirajućom smolom i uretan-dimetakrilatnom smolom u crnom vinu, koli i kavi kao medijima za pohranu, jer su to često konzumirani napitci. Testirani materijali za izradu baze proteze pokazali su značajno različitu diskoloraciju nakon pohranjivanja u različitim medijima u objema fazama ocjenjivanja. Zato je nulta hipoteza istraživanja odbačena.

$\mathrm{U}$ dentalnoj medicini vidljiva promjena boje $(\Delta \mathrm{E}>1)$ smatra se prihvatljivom do vrijednosti od $\Delta \mathrm{E}=3,3$; razlika iznad toga je neprihvatljiva $(24,25)$. Samo skupina materijala Eclypse pohranjena u crnom vinu pokazala je klinički neprihvatljivu promjenu boje nakon 30 dana $(\Delta \mathrm{E} 3,59)$.

Utvrdena je promjena boje svih akrilata za proteze i sve su se s vremenom povećavale. Postoje unutarnji i vanjski čimbenici koji mogu uzrokovati promjenu boje materijala za izradu baze proteze $(15,26)$. To su fizikalno-kemijske promjene, nakupljanje mrlja, zaostatni monomer, upijanje vode i razgradnja unutarnjih pigmenata, otapanje sastojaka i hrapavost površine. Poznato je da napitci poput kave i kole te crno vino uzrokuju promjenu boje svih smola za izradu baze proteze $(18,22,26)$. 
coke and red wine enhance the discoloration of all denture base resins $(18,22,26)$.

Zuo et al. examined the discoloration of different denture base resins after immersion in different cleaners and different beverages (26). The conclusion of the study points to the fact that color change of the Eclipse denture base resin was much higher than the clinically acceptable value of $\Delta \mathrm{E} 3.3$. This is in line with the present study results which showed that the Eclipse denture base groups had the most defined color change. This result could be due to the tendency of high water absorption in light activated denture base materials when compared to the other materials. Kerby et al. reported that Eclipse is also sensitive to hygroscopic expansion; this is caused by the 2 hydrophilic urethane groups within its molecular structure, but less than PMMA (27). Further studies, which take both water absorption and color change of different denture base materials into account, are needed.

CAD/CAM fabricated acrylic has achieved a better color stability, better mechanical properties, prevention of porosities and a better fit than the conventional PMMA resins. Polymerization methods and composition of a resin matrix may have a great effect on its stability of color (28-30). Conventionally fabricated PMMA resins are dependent on the technician, mixing proportions of the resin components, polymerization device and duration of the polymerization, among others (31). According to the results of the present study, the least color change was observed in CAD-CAM denture base resins in all of the beverages. This is due to the fact that it can be better polished, there is no porous structure, less water absorption and less wear as proved in the literature.

Alp et al. examined the effect of coffee solutions on the discoloration of different CAD-CAM acrylic resins, likewise the current study, researchers reported that clinically admissible color changes did not occur in different denture base acrylic resins due to coffee staining (32). However, researchers observed that the color change and surface roughness in heat-polymerized and different pre-polymerized CAD-CAM PMMA specimens were not significantly different. This may be attributed to the 8 hours of heat polymerization of the heat-polymerized control group, which enhance its physical features.

Presently, there are varieties of options available including the new generation of PMMA-based self-polymerizing denture base materials. These materials have a shorter production process but the residual monomer can cause an enhance risk of tissue reactions and it has decreased mechanical properties (33). According to a previous study, Weropress have acceptable flexural properties when compared to traditional heat polymerized resins and light-activated resins (34). The present study reported that Weropress had a clinically acceptable color change and this material needs further study in order to determine residual monomer degree and cytotoxicity.

In the previous study, similar to other studies, a minimum color change was detected in the specimens which were immersed in distilled water $(1,35)$. The reason for this situation is that there are no substances which may cause discoloration in materials, and $\mathrm{pH}$ of the distilled water does not cause roughness on the surface due to neutrality.
Zuo i suradnici ispitali su promjenu boje različitih smola za izradu baze proteze nakon uranjanja u različita sredstva za čišćenje i različita pića (26). Zaključak istraživanja pokazao je da je promjena boje smole Eclypsea znatno veća od klinički prihvatljive vrijednosti $\Delta \mathrm{E} 3,3$. To je u skladu s rezultatima ovog istraživanja koji su pokazali da je Eclypse najviše promijenio boju. Taj rezultat može biti posljedica sklonosti prema apsorpciji vode materijala za bazu proteze koji se svjetlosno polimeriziraju u usporedbi s drugim materijalima. Kerby i suradnici izvijestili su da je Eclypse također osjetljiv na higroskopno širenje; to je uzrokovano dvjema hidrofilnim uretanskim skupinama unutar njegove molekularne strukture, ali manje od PMMA-e (27). Potrebna su daljnja istraživanja koja će uzeti u obzir i apsorpciju vode i promjenu boje različith materijala za izradu baze proteze.

Akrilati obrađeni CAD/CAM tehnologijom postižu bolju stabilnost boje i bolja mehanička svojstva, manje su porozni i imaju bolji dosjed u odnosu prema konvencionalnim PMMA smolama. Metode polimerizacije i sastav smolaste matrice mogu znatno utjecati na stabilnost boje $(28-30)$. Konvencionalno proizvedene PMMA smole, između ostaloga, ovise o tehničaru, omjeru miješanja komponenti smole, polimerizacijskom uređaju i trajanju polimerizacije (31). Prema rezultatima ovog istraživanja, najmanja promjena boje nakon pohranjivanja u svim napitcima, zabilježena je za $\mathrm{CAD} / \mathrm{CAM}$ materijal za izradu baze proteze. To je zato što se može bolje polirati, nema poroznu strukturu, manje upija vodu i manje se troši, kao što je dokazano u literaturi.

Alp i suradnici ispitali su utjecaj kave na diskoloraciju različitih CAD/CAM akrilatnih smola i, kao i u ovom istraživanju, izvijestili da se klinički prihvatljive promjene boje nisu pojavile na različitim akrilatnim smolama za izradu baze proteze nakon uranjanja u kavu (32). No uočili su da se promjena boje i hrapavost površine toplinski polimerizirajućih i različitih pretpolimeriziranih CAD/CAM PMMA uzoraka nisu značajno razlikovale. To se može pripisati osmosatnoj toplinskoj polimerizaciji uzoraka kontrolne skupine, što poboljšava njihova fizička svojstva.

Trenutačno postoje razne mogućnosti, uključujući novu generaciju autopolimerizirajućih materijala za izradu proteza na bazi PMMA-e. Ti materijali imaju kraći proizvodni proces, ali rezidualni monomer uzrokuje povećan rizik od reakcija tkiva i lošija su im mehanička svojstva (33). Prema ranije provedenom istraživanju, Weropress ima prihvatljiva svojstva savijanja u usporedbi $s$ tradicionalnim toplinski i svjetlosno polimerizirajućim smolama (34). To istraživanje pokazalo je da Weropress klinički prihvatljivo mijenja boju i da je za taj materijal potrebno dodatno analizirati stupanj rezidualnog monomera i citotoksičnost.

U jednoj ranijoj studiji, slično drugim istraživanjima, minimalna promjena boje otkrivena je u uzorcima koji su uronjeni u destiliranu vodu $(1,35)$. Razlog za to jest što u njoj ne postoje tvari koje mogu uzrokovati promjenu boje na materijalu, a pH destilirane vode ne uzrokuje hrapavost površine zbog neutralnosti.

Dokazano je da sadržaj alkohola u napitcima uzrokuje degradaciju površine i ekspanziju, a time i lošija fizikalna svojstva smole. Učinak bojenja crvenog vina može biti uzrokovan 
Coloring effects of red wine can be caused by its alcohol content that causes denture base surfaces to become rough (35). In the current study, a higher color difference for all denture base resins was observed in the red wine group than the coke or coffee group. Although red wine has a relatively low acidic $\mathrm{pH}$ (3.6) when compared to coke, it still caused greater discoloration.

Other explanations of the coloring effects of red wine may be the softening of the materials from the absorption of alcohol molecules into the organic matrix and following change in surface smoothness (36-38). The most probable cause is that the acidic $\mathrm{pH}$ and alcohol content of red wine affected the surface roughness of the prosthetic base. Red wine contains anthocyanin that is a water-soluble pigment which provides the grapes their color ${ }^{39,40}$. The color change of the resin denture base which was stored in red wine was presumably a result of the concentration of the red color coming from its pigments, along with the higher absorption of the red pigments due to alcohol, which has a plasticizing effect on the organic matrix during the storage period, thus causing a significant color change in the denture base.

The present study has a number of limitations. First, the present in vitro result prerequisites need to be tested in vivo trials. However, in vivo studies are more challenging to be carried out. Besides, standardization in vivo studies is less likely to be accomplished using the methods applied in the current study. Although, the discoloration of denture base materials evaluated in vitro methods may not be as accurate or valid as those obtained through in vivo methods, they can provide useful guidance for clinical applications. Despite the limitations of the current study it presents beneficial evidence regarding the color change of recently introduced CAD-CAM denture base resins and the discoloration of some beverages, which are frequently used up in daily life.

\section{Conclusion}

Within the limitations of this in vitro study, the following conclusions could be drawn: The color stability of CADCAM denture base resins is better than that of some other kinds of denture base resins. All the changes in the color values of the groups, except those in Eclypse which was stored in red wine, were under the clinically perceptible value. The color stability of the Eclipse denture base resin was lower compared to other denture base groups. All beverages used in the study had an effect on color change.

\section{Conflict of interest}

The authors report no conflict of interest. sadržajem alkohola koji čini da površina baze proteze postane hrapava (35). U ovom istraživanju najveće razlike u boji za sve smole za izradu baze proteze, zabilježene su u skupini s crnim vinom, kolom ili kavom. Iako crno vino ima razmjerno nizak pH $(3,6)$ u usporedbi s kolom, ipak je uzrokovalo veću diskoloraciju.

Drugo objašnjenje učinka bojenja crvenog vina može biti omekšavanje materijala zbog apsorpcije molekula alkohola u organsku matricu i posljedične promjene u glatkoći površine (36 - 38). Najvjerojatniji uzrok je da kiseli pH i sadržaj alkohola u crnom vinu utječu na hrapavost površine baze proteze. Crveno vino sadržava antocijanin, pigment topljiv u vodi koji grožđu daje boju $(39,40)$. Promjena boje baze zubne proteze koja je pohranjena u crvenom vinu vjerojatno je posljedica koncentracije crvene boje koja potječe od njegovih pigmenata, zajedno $s$ većom apsorpcijom crvenih pigmenata zbog alkohola koji plastificirajuće djeluje na organsku matricu tijekom pohranjivanja te tako uzrokuje značajnu promjenu boje baze proteze.

Ovo istraživanje imalo je niz ograničenja. Istaknimo da dobivene rezultate in vitro treba testirati u uvjetima in vivo. No istraživanja in vivo teže se provode i ne mogu se postići standardizirane metode primijenjene u ovom istraživanju. Premda procjena promjene boje materijala metodama in vitro možda nije toliko precizna ili valjana kao ona dobivena metodama in vivo, može pružiti korisne smjernice za kliničku primjenu. Unatoč ograničenjima u ovom istraživanju, dobiveni su korisni dokazi o promjeni boje suvremenih CAD/CAM materijala za izradu baze proteze i o diskoloraciji nekih napitaka koji se često konzumiraju.

\section{Zaključak}

Uzimajući u obzir ograničenja ovog istraživanja in vitro, može se zaključiti sljedeće: stabilnost boje CAD/CAM materijala za izradu baze proteze bila je bolja od ostalih smola; sve promjene boje u skupinama, osim za Eclypse koji je pohranjen u crvenom vinu, bile su klinički vidljive; stabilnost boje materijala Eclypse bila je niža od ostalih skupina materijala za bazu proteze; sva pića korištena u istraživanju utjecala su na promjenu boje.

\section{Sukob interesa}

Autori nisu bili u sukobu interesa. 
Sažetak

Cilj: Željela se procijeniti stabilnost boje toplinski polimerizirajuće smole, autopolimerizirajuće smole, uretan-dimetakrilatne smole i CAD/CAM bloka od polimetilmetakrilata (PMMA) pohranjenih u različitim medijima. Materijali i metode: Za svaku skupinu proizvedeno je 60 uzoraka u obliku pločica $(15 \times 2 \mathrm{~mm})$. Ukupno 240 uzoraka termociklirano je u 5000 ciklusa. Zatim su uzorci slučajnim odabirom podijeljeni u četiri skupine $(n=15)$ prema mediju za pohranu: kava, kola, crno vino i destilirana voda (kontrolna skupina). Mjerenje boje svakog uzorka obavljeno je spektrofotometrom prije pohranjivanja i poslije toga postupka (nakon 7 i 30 dana) te su izračunate razlike u boji (ĆE). Rezultati: U svim materijalima za bazu proteze dogodile su se promjene boje različitog stupnja nakon pohranjivanja u različitim medijima tijekom obaju mjerenja. U svim medijima za pohranjivanje CAD/CAM materijali minimalno su promijenili boju. U svim materijalima za izradu baze proteze crno vino uzrokovalo je veći stupanj promjene boje od kole ili kave. Zaključak: Stabilnost boje CAD/CAM materijala za izradu baze proteze bila je bolja od bilo koje druge vrste materijala. Vrijednosti promjene boje u svim skupinama pohranjenima u crnom vinu bile su klinički vidljive (osim Eclypsea).
Zaprimljen: 28. ožujka 2019.

Prihvaćen: 1. lipnja 2019.

Adresa za dopisivanje

Cagatay Dayan

Istanbul Cerrahpasa University

Program of Dental Technicians

Istanbul, Turkey

suleyman.dayan@istanbul.edu.tr

Ključne riječi

PMMA, CAD/CAM akrilatni blok, diskoloracija, materijal za izradu baze proteze

\section{References}

1. Sepúlveda-Navarro WF, Arana-Correa BE, Borges CP, Jorge JH, Urban VM, Campanha NH. Color stability of resins and nylon as denture base material in beverages. J Prosthodont. 2011; 20:632-8.

2. Goiato MC, Nóbrega AS, dos Santos DM, Andreotti AM, Moreno A. Effect of different solutions on color stability of acrylic resinbased dentures. Braz Oral Res. 2014; 1:1-7.

3. Tuna EB, Rohlig BG, Sancakli E, Evlioglu G, Gencay K. Influence of acrylic resin polymerization methods on residual monomer release. J Contemp Dent Pract. 2013 Mar 1;14(2):259-64.

4. Choi JE, Ng TE, Leong CKY, Kim H, Li P, Waddell JN. Adhesive evaluation of three types of resilient denture liners bonded to heatpolymerized, autopolymerized, or CAD-CAM acrylic resin denture bases. J Prosthet Dent. 2018 Nov;120(5):699-705.

5. Berger JC, Driscoll CF, Romberg E, Luo Q, Thompson G. Surface roughness of denture base acrylic resins after processing and after polishing. J Prosthodont. 2006 May-Jun;15(3):180-6.

6. Gungor H, Gundogdu M, Yesil Duymus Z. Investigation of the effect of different polishing techniques on the surface roughness of denture base and repair materials. J Prosthet Dent. 2014; 112:1271-7.

7. Singh S, Palaskar JN, Mittal S. Comparative evaluation of surface porosities in conventional heat polymerized acrylic resincured by water bath and microwave energy with microwavable acrylic resin cured by microwave energy. Contemp Clin Dent. 2013; 4:147-51.

8. Machado C, Sanchez E, Azer SS, Uribe JM. Comparative study of the transverse strength of three denture base materials. J Dent. 2007 Dec;35(12):930-3.

9. Alp G, Murat S, Yilmaz B. Comparision of flexural strength of different CAD/CAM PMMA-based polymers. J Prosthodont. 2019 Feb;28(2):e491-e495.

10. Steinmassl PA, Wiedemair V, Huck C, Klaunzer F, Steinmassl O, Grunert I, Dumfahrt H. Do CAD/CAM dentures really release less monomer than conventional dentures? Clinical oral investigations. 2017;21:1697-705.

11. Güth JF, Almeida E Silva JS, Beuer F F, Edelhoff D. Enhancing the predictability of complex rehabilitation with a removable CAD/ CAM-fabricated long-term provisional prosthesis: a clinical report. J Prosthet Dent. 2012 Jan;107(1):1-6.

12. Edelhoff D, Beuer F, Schweiger J, Brix O, Stimmelmayr M, Guth JF. CAD/CAM-generated high-density polymer restorations for the pretreatment of complex cases: a case report. Quintessence Int. 2012 Jun;43(6):457-67.

13. Oğuz S, Mutluay MM, Doğan OM, Bek B. Color change evaluation of denture soft lining materials in coffee and tea. Dent Mater J. 2007;26:209-16.

14. Waliszewski M. Restoring dentate appearance: a literature review for modern complete denture esthetics. J Prosthet Dent. 2005;93:386-94.

15. Goiato MC, Santos DM, Haddad MF, Pesqueira AA. Effect of accelerated aging on the microhardness and color stability of flexible resins for dentures. Braz Oral Res. 2010; 24:114-9.

16. Heydecke G, Locker D, Awad MA, Lund JP, Feine JS. Oral and general health-related quality of life with conventional and implant dentures. Community Dent Oral Epidemiol. 2003 Jun;31(3):161 8.

17. Sahin O, Koroglu A, Dede DÖ, Yilmaz B. Effect of surface sealant agents on the surface roughness and color stability of denture base materials. J Prosthet Dent. 2016; 116:610-616.
18. Hong G, Murata H, Li Y, Sadamori S, Hamada T. Influence of denture cleansers on the color stability of three types of denture base acrylic resin. J Prosthet Dent. 2009; 101:205-13.

19. Goiato MC, Nóbrega AS, dos Santos DM, Andreotti AM, Moreno A. Effect of different solutions on color stability of acrylic resinbased dentures. Braz Oral Res. 2014;1:1-7.

20. Moon A, Powers JM, Kiat-Amnuay S. Color stability of denture teeth and acrylic base resin subjected daily to various consumer cleansers. J Esthet Restor Dent. 2014 Jul-Aug;26(4):247-55.

21. Rutkunas V, Sabaliauskas V, Mizutani H. Effects of different food colorants and polishing techniques on color stability of provisional prosthetic materials. Dent Mater J. 2010 Mar;29(2):167-76.

22. Imirzalioglu P, Karacaer O, Yilmaz B, Ozmen Msc I. Color stability of denture acrylic resins and a soft lining material against tea, coffee, and nicotine. J Prosthodont. 2010; 19:118-24.

23. Steinmassl PA, Klaunzer F, Steinmassl O, Dumfahrt H, Grunert I. Evaluation of Currently Available CAD/CAM Denture Systems. Int J Prosthodont. 2017 Mar/Apr;30(2):116-122.

24. Goiato MC, Dos Santos DM, Baptista GT, Moreno A, Andreotti AM, Bannwart LC, Dekon SF. Effect of thermal cycling and disinfection on colour stability of denture base acrylic resin. Gerodontology. 2013 Dec;30(4):276-82.

25. Hollis S, Eisenbeisz E, Versluis A. Color stability of denture resins after staining and exposure to cleansing agents. J Prosthet Dent. 2015;114:709-14.

26. Zuo W, Feng D, Song A, Gong H, Zhu S. Effects of organic-inorganic hybrid coating on the color stability of denture base resins. J Prosthet Dent. 2016;115:103-8.

27. Kerby RE, Knobloch LA, Schricker S, Gregg B. Synthesis and evalu ation of modified urethane dimethacrylate resins with reduced water sorption and solubility. Dent Mater. 2009 Mar;25(3):302-13.

28. Rayyan MM, Aboushelib M, Sayed NM, Ibrahim A, Jimbo R. Comparison of interim restorations fabricated by CAD/CAM with those fabricated manually. J Prosthet Dent. 2015; 114:414-9.

29. Yao J, Li J, Wang Y, Huang H. Comparison of the flexural strength and marginal accuracy of traditional and CAD/CAM interim materials before and after thermal cycling. J Prosthet Dent. 2014 Sep;112(3):649-57.

30. Alt V, Hannig M, Wostmann B, Balkenhol M. Fracture strength of temporary fixed partial dentures: CAD/CAM versus directly fabricated restorations. Dent Mater. 2011 Apr;27(4):339-47.

31. Stawarczyk B, Ender A, Trottmann A, Özcan M, Fischer J, Hämmer le $\mathrm{CH}$. Load-bearing capacity of CAD/CAM milled polymeric threeunit fixed dental prostheses: effect of aging regimens. Clin Oral Investig. 2012; 16:1669-77.

32. Alp G, Johnston WM, Yilmaz B. Optical properties and surface roughness of prepolymerized poly(methyl methacrylate) denture base materials. J Prosthet Dent. 2019 Feb;121(2):347-352.

33. Pfeiffer P, Rosenbauer EU. Residual methyl methacrylate monomer, water sorption, and water solubility of hypoallergenic denture base materials. J Prosthet Dent. 2004;92:72-78.

34. Mumcu E, Cilingir A, Gencel B, Sülün T. Flexural properties of a light-cure and a self-cure denture base materials compared to conventional alternatives. J Adv Prosthodont. 2011 Sep;3(3):136 9.

35. Erdemir U, Kaner AO, Eren MM, Ozan G, Yıldız E. Color stability of bulk-fill composites immersed in different drinks. Color Research \& Application. 2018; 43:785-93. NIJE U PUBMEDU 
36. Ceci M, Viola M, Rattalino D, Beltrami R, Colombo M, Poggio C. Discol- oration of different esthetic restorative materials: a spectrophotometric eval- uation. Eur J Dent. 2017; 11:149-56.

37. Catelan A, Briso AL, Sundfeld RH, Goiato MC, dos Santos PH. Color stability of sealed composite resin restorative materials after ultraviolet arti- ficial aging and immersion in staining solutions. J Prosthet Dent. 2011;105:236-41.

38. Omata Y, Uno S, Nakaoki Y, Tanaka T, Sano H, Yoshida S, Sidhu SK. Staining of hybrid composites with cof- fee, oolong tea, or red wine. Dent Mater J. 2006 Mar;25(1):125-31.
39. Soares-Geraldo D, Scaramucci T, Steagall-Jr W, Braga SR, Sobral MA. Interaction between staining and degradation of a composite resin in contact with colored foods. Braz Oral Res. 2011;25:36975.

40. Tanthanuch S, Kukiattrakoon B, Peerasukprasert T, Chanmanee $\mathrm{N}$, Chaisomboonphun P, Rodklai A. The effect of red and white wine on color changes of nanofilled and nanohybrid resin composites. Restor Dent Endod. 2016 May;41(2):130-6. 